VOL. 73 (2006) [89-105]

\title{
ANALOGUES OF THE LIOUVILLE THEOREM FOR LINEAR FRACTIONAL RELATIONS IN BANACH SPACES
}

\author{
V.A. KhatskeVich, M.I. Ostrovskil and V.S. Shulman
}

Consider a bounded linear operator $T$ between Banach spaces $\mathcal{B}, \mathcal{B}^{\prime}$ which can be decomposed into direct sums $\mathcal{B}=\mathcal{B}_{1} \oplus \mathcal{B}_{2}, \mathcal{B}^{\prime}=\mathcal{B}_{1}^{\prime} \oplus \mathcal{B}_{2}^{\prime}$. Such linear operator can be represented by a $2 \times 2$ operator matrix of the form

$$
T=\left(\begin{array}{ll}
T_{11} & T_{12} \\
T_{21} & T_{22}
\end{array}\right),
$$

where $T_{i j} \in \mathcal{L}\left(\mathcal{B}_{j}, \mathcal{B}_{i}^{\prime}\right), i, j=1,2$. (By $\mathcal{L}\left(\mathcal{B}_{j}, \mathcal{B}_{i}^{\prime}\right)$ we denote the space of bounded linear operators acting from $\mathcal{B}_{j}$ to $B_{i}^{\prime}(i, j=1,2)$.) The map $G_{T}$ from $\mathcal{L}\left(\mathcal{B}_{1}, \mathcal{B}_{2}\right)$ into the set of closed affine subspaces of $\mathcal{L}\left(\mathcal{B}_{1}^{i}, B_{2}^{\prime}\right)$, defined by

$$
G_{T}(X)=\left\{Y \in \mathcal{L}\left(\mathcal{B}_{1}^{\prime}, \mathcal{B}_{2}^{\prime}\right): T_{21}+T_{22} X=Y\left(T_{11}+T_{12} X\right)\right\}
$$

is called a linear fractional relation associated with $T$.

Such relations can be considered as a generalisation of linear fractional transformations which were studied by many authors and found many applications. Many traditional and recently discovered areas of application of linear fractional transformations would benefit from a better understanding of the behaviour of linear fractional relations. The present paper is devoted to analogues of the Liouville theorem "a bounded entire function is constant" for linear fractional relations.

\section{INTRODUCTION}

The subject of the present paper belongs to both linear and non-linear analysis: it considers some problems of infinite-dimensional holomorphic analysis of multivalued non-linear maps, constructed via linear bounded operators between Banach spaces.

Consider a bounded linear operator $T$ between Banach spaces $\mathcal{B}, \mathcal{B}^{\prime}$ which can be decomposed into direct sums $\mathcal{B}=\mathcal{B}_{1} \oplus \mathcal{B}_{2}, \mathcal{B}^{\prime}=\mathcal{B}_{1}^{\prime} \oplus \mathcal{B}_{2}^{\prime}$. Such linear operator can be represented by a $2 \times 2$ operator matrix of the form

$$
T=\left(\begin{array}{ll}
T_{11} & T_{12} \\
T_{21} & T_{22}
\end{array}\right)
$$

Received 4th October, 2005

Copyright Clearance Centre, Inc. Serial-fee code: 0004-9727/06 \$A2.00+0.00. 
where $T_{i j} \in \mathcal{L}\left(\mathcal{B}_{j}, \mathcal{B}_{i}^{\prime}\right), i, j=1,2$. (By $\mathcal{L}\left(\mathcal{B}_{j}, \mathcal{B}_{i}^{\prime}\right)$ we denote the space of bounded linear operators acting from $\mathcal{B}_{j}$ to $\mathcal{B}_{i}^{\prime}(i, j=1,2)$.

With each such matrix $T$ one can associate a map (defined on some, possibly empty, part of $\left.\mathcal{L}\left(\mathcal{B}_{1}, \mathcal{B}_{2}\right)\right)$ by the formula

$$
H_{T}(X) \doteq\left(T_{21}+T_{22} X\right)\left(T_{11}+T_{12} X\right)^{-1} .
$$

Such maps are called (operator) linear fractional transformations.

Krein $[16,17]$ discovered that linear fractional transformations can serve as a powerful tool in the study of operators on indefinite metric spaces. The theory of linear fractional transformations, with their comparatively simple algebraic and complicated analytic properties, is an interesting subject of investigation which attracted many prominent mathematicians (Helton, Iokhvidov, Krein, Langer, Shmulian, and others, see $[2,7,8,18,19,20])$. Operator linear fractional transformations found applications in non-linear holomorphic analysis in Banach spaces (see, for example, $[3,4,6,15]$ and references therein), to Koenigs embedding problem, Abel-Schröder equations, composition operators on Hardy and Bergman spaces, theory of generators of non-linear semigroups, and to many other problems (see $[1,3,4,6,13,14,22]$, and references therein). In most of these applications the requirement that $\left(T_{11}+T_{12} X\right)$ is invertible (needed to define a linear fractional transformation) is not natural and is quite restrictive. In this connection it became important to generalise results of the theory of linear fractional transformations to the case when $\left(T_{11}+T_{12} X\right)$ is not invertible. Work in this direction has been done in $[9,10,11,12]$. In these papers results of the theory of linear fractional transformations were generalised through the study of multivalued maps defined in the following way.

Definition 1: The map $G_{T}$ from $\mathcal{L}\left(\mathcal{B}_{1}, \mathcal{B}_{2}\right)$ into the set of closed affine subspaces of $\mathcal{L}\left(B_{1}^{\prime}, \mathcal{B}_{2}^{\prime}\right)$, defined by

$$
G_{T}(X)=\left\{Y \in \mathcal{L}\left(\mathcal{B}_{1}^{\prime}, \mathcal{B}_{2}^{\prime}\right): T_{21}+T_{22} X=Y\left(T_{11}+T_{12} X\right)\right\},
$$

is called a linear fractional relation (associated with $T$ ).

Definition 2: A linear fractional relation $G_{T}$ is said to be defined at $X$ if $G_{T}(X)$ $\neq \emptyset$. The set of all $X \in \mathcal{L}\left(\mathcal{B}_{1}, \mathcal{B}_{2}\right)$ at which $G_{T}$ is defined is called the domain of $G_{T}$ and is denoted by $\operatorname{dom} G_{T}$.

An interesting (though somewhat vague) problem is: how should one define and check "holomorphic" properties of multivalued maps? In this paper we mainly consider, for linear fractional relations (which without doubt can be considered as "holomorphic" multivalued maps and expected to have the corresponding behaviour) one such property - the validity of analogues of Liouville's theorem "a bounded entire function is constant". Clearly for single-valued holomorphic (in any reasonable sense) maps between Banach 
spaces the direct analogue is valid. In the multivalued case one should first define constant and bounded maps. We use the following definitions. Let $G$ be a mutivalued map from an arbitrary set $S$ into a Banach space $B$. We say that $G$ is constant on a subset $U$ of $S$ if there is an element $y$ belonging to $G(s)$ for all $s \in U$. The map $G$ is called bounded on $U$ if there is $C>0$ such that $\inf _{y \in G(s)}\|y\| \leqslant C$ for each $s \in U$.

It was noted in [12] that even in the case when $\mathcal{B}$ and $\mathcal{B}^{\prime}$ are Hilbert spaces, there are non-constant and non-linear linear fractional relations defined on the whole $\mathcal{L}\left(\mathcal{B}_{1}, \mathcal{B}_{2}\right)$. We shall give such an example for the convenience of the reader, because in [12] it was not clearly written out.

Let $T_{11}$ be an isometry with the image $Z \subset \mathcal{B}_{1}^{\prime}$ of infinite codimension. Let $T_{12}$ be a compact operator whose image is orthogonal to $Z$. Operators $T_{2 i}$ can be arbitrary. Then, for each $X$, the operator $T_{11}+T_{12} X$ has trivial kernel and a closed image. Since $\mathcal{B}_{1}$ is a Hilbert space, it follows that $G_{T}(X)$ is non-empty. The map $G_{T}$ is clearly non-constant if, for example, $T_{22}$ is a non-compact operator.

We are going to study the following problem on analogues of the Liouville theorem for linear fractional relations: can maps $G_{T}$ be defined and bounded on $\mathcal{L}\left(\mathcal{B}_{1}, \mathcal{B}_{2}\right)$ without being constant? The answer is surprising: it depends on the geometry of the spaces. Namely, for reflexive spaces the answer is negative (Theorem 1), but in general the answer is affirmative (Theorem 2).

Theorem 1 actually proves (for spaces complemented in their second duals) much more than an analogue of the Liouville theorem: if a linear fractional transformation is bounded on its domain, then it is constant. For linear fractional transformations this "strong Liouville theorem" (without any restrictions on the Banach spaces involved) will be established in Theorem 3 .

In the last section of the work we consider similar problems for Banach (mostly $C^{*}$-) algebras.

\section{Preliminary RESUlts}

For linear fractional relations the notion of constant maps introduced above can be written in the following way.

Definition 3: A linear fractional relation $G_{T}$ is called constant on $A \subset \mathcal{L}\left(\mathcal{B}_{1}, \mathcal{B}_{2}\right)$ if $A \subset \operatorname{dom} G_{T}$ and there exists $W \in \mathcal{L}\left(\mathcal{B}_{1}^{\prime}, \mathcal{B}_{2}^{\prime}\right)$ such that $W \in G_{T}(X)$ for each $X \in A$. If $G_{T}$ is constant on $\mathcal{L}\left(\mathcal{B}_{1}, \mathcal{B}_{2}\right)$ we simply say that $G_{T}$ is constant.

It turns out that if $G_{T}$ is constant on a rich set (see the definition below), then the second row of $T$ is an operator multiple of the first row (see (ii) in Proposition 1). For Hilbert space operators this was observed in [12]. Below we show that the same argument works for Banach space operators. 
Definition 4: A subset $A$ of $\mathcal{L}\left(\mathcal{B}_{1}, \mathcal{B}_{2}\right)$ is called rich if the subspace of $B_{2}$ spanned by the union of all subspaces of the form $\left(K_{1}-K_{2}\right)\left(B_{1}\right)$, where $K_{1}, K_{2} \in A$, is dense in $\boldsymbol{B}_{2}$.

Proposition 1. Let $A \subset \mathcal{L}\left(\mathcal{B}_{1}, \mathcal{B}_{2}\right)$ be rich. For a matrix $T$ the following conditions are equivalent:

(i) $G_{T}$ is constant on $A$.

(ii) There exists an operator $W$ in $\mathcal{L}\left(\mathcal{B}_{1}^{\prime}, \mathcal{B}_{2}^{\prime}\right)$ such that

$$
T=\left(\begin{array}{cc}
T_{11} & T_{12} \\
W T_{11} & W T_{12}
\end{array}\right)
$$

(iii) $G_{T}$ is constant.

Proof: We start by proving (i) $\Rightarrow$ (ii). Let $G_{T}$ be constant on a rich set $A$ and let $W$ be an operator satisfying

$$
W \in G_{T}(K) \quad \forall K \in A .
$$

Let $K_{1}, K_{2} \in A$. Then

$$
T_{21}+T_{22} K_{1}=W\left(T_{11}+T_{12} K_{1}\right)
$$

and

$$
T_{21}+T_{22} K_{2}=W\left(T_{11}+T_{12} K_{2}\right)
$$

Subtracting (6) from (5) we get

$$
T_{22}\left(K_{1}-K_{2}\right)=W T_{12}\left(K_{1}-K_{2}\right) .
$$

Therefore $T_{22} x=W T_{12} x$ for each $x \in\left(K_{1}-K_{2}\right)\left(\mathcal{B}_{1}\right)$. Since $A$ is rich, it implies $T_{22}=W T_{12}$.

Now we derive $T_{21}=W T_{11}$ from either (5) or (6).

The implications (ii) $\Rightarrow$ (iii) $\Rightarrow$ (i) are obvious.

REMARK. In general, if $G_{T}$ is constant on its domain, it does not imply that the second row of $T$ is a multiple of the first. As we shall see in Theorem 1 , the only example of this kind is

$$
T=\left(\begin{array}{cc}
0 & 0 \\
0 & T_{22}
\end{array}\right)
$$

with $T_{22} \neq 0$. 


\section{MAIN RESULTS}

In this section we find analogues of the Liouville Theorem for linear fractional relation. The restriction of the general definition of boundedness (mentioned above) to the case of linear fraction relations is:

Definition 5: A linear fractional relations $G_{T}$ is called bounded if

$$
\sup _{x \in \operatorname{dom}\left(G_{T}\right)} \inf _{Y \in G_{T}(X)}\|Y\|<\infty .
$$

Our first result is the following analogue of the Liouville Theorem for linear fraction relations between Banach space operators.

THEOREM 1. Let $\mathcal{B}_{2}^{\prime}$ be such that the canonical image of $\mathcal{B}_{2}^{\prime}$ is complemented in $\left(\mathcal{B}_{2}^{\prime}\right)^{* *}$. If $\operatorname{dom} G_{T} \neq \emptyset$ and $G_{T}$ is bounded, then either $G_{T}$ is constant, or $T$ is of the form

$$
T=\left(\begin{array}{cc}
0 & 0 \\
0 & T_{22}
\end{array}\right)
$$

with $T_{22} \neq 0$. In the latter case $G_{T}$ is defined at $X$ if and only if $T_{22} X=0$, and, for such $X, G_{T}(X)=\mathcal{L}\left(\mathcal{B}_{1}^{\prime}, \mathcal{B}_{2}^{\prime}\right)$. Therefore in both cases $G_{T}$ is constant on $\operatorname{dom} G_{T}$.

Proof: First we prove the theorem in the case $T_{21}=0$. In such a case $0 \in \operatorname{dom} G_{T}$, $0 \in G_{T}(0)$, and the equation in (3) becomes

$$
T_{22} X=Y\left(T_{11}+T_{12} X\right)
$$

Our first purpose is to prove that in this case $\operatorname{dom} G_{T}$ contains all operators of finite rank. Let $R$ be the set of operators $X$ of rank one in $\operatorname{dom} G_{T}$, such that $G_{T}(X)$ contains a rank one operator. An operator $X=u \otimes f$ (this means that $X x=f(x) u$ for all $x$ ) belongs to $R$ if and only if there is an operator $Y=p \otimes g$ satisfying (8). This condition can be written as

$$
p \otimes\left(T_{11}^{*} g+g\left(T_{12} u\right) \cdot f\right)=T_{22} u \otimes f
$$

It is clear that this condition is satisfied for arbitrary $u$ and $g$ satisfying $g\left(T_{12} u\right) \neq 1$, if we let $f=\left(1-g\left(T_{12} u\right)\right)^{-1} T_{11}^{*} g$ and $p=T_{22} u$. In particular, the condition (9) is satisfied if $g\left(T_{12} u\right)=0, f=T_{11}^{*} g$, and $p=T_{22} u$.

For $x \in \mathcal{B}_{1}$ we denote by $W(x)$ the set of all vectors $y \in \mathcal{B}_{2}$ which can be written in the form $y=X x$, for some $X \in R$.

CASE 1. $T_{11} \neq 0$. Suppose that $T_{11} x \neq 0$. Let $X=u \otimes f$, we have $X x=f(x) u$. Take $g_{0} \in\left(\mathcal{B}_{1}^{\prime}\right)^{*}$ with $g_{0}\left(T_{11} x\right) \neq 0, g=\lambda g_{0}$, and $f=\left(\lambda /\left(1-\lambda g\left(T_{12} u\right)\right)\right) T_{11}^{*} g_{0}$. Then

$$
X x=\frac{\lambda}{1-\lambda g\left(T_{12} u\right)} \cdot g_{0}\left(T_{11} x\right) u
$$


can be arbitrarily close to $u$ (if one choose $\lambda$ in a proper way). This means that $W(x)$ is dense in $\mathcal{B}_{2}$ if $T_{11} x \neq 0$. Therefore for $T_{11} \neq 0$ the set $Q$ of all pairs $(x, y)$ satisfying $y \in W(x)$ is dense in $\mathcal{B}_{1} \times \mathcal{B}_{2}$.

Let

$$
C=\sup _{X \in \operatorname{dom} G_{T}} \inf _{Y \in G_{T}(X)}\|Y\| .
$$

Then

$$
\left\|T_{22} X x\right\| \leqslant C\left\|\left(T_{11}+T_{12} X\right) x\right\|
$$

for all $X \in R$ and all $x$. Hence

$$
\left\|T_{22} y\right\| \leqslant C\left\|T_{11} x+T_{12} y\right\|
$$

for all $(x, y) \in Q$. Since $Q$ is dense, we may assume that (11) holds for all $x, y$.

In particular, the inequality (10) holds for each $X$ and all $x$. So, for a fixed finite rank operator $X$, setting $Y\left(T_{11}+T_{12} X\right) x=T_{22} X x$ we define a bounded operator $Y$ on the linear subspace $\left(T_{11}+T_{12} X\right) \mathcal{B}_{1}$. Being finite rank it extends to whole $\mathcal{B}_{2}$ and clearly belongs to $G_{T}(X)$. Thus dom $G_{T}$ contains all operators of finite rank.

Now we show that for each triple $(M, N, \varepsilon)$, where $M$ and $N$ are finite dimensional subspaces in $\mathcal{B}_{1}$ and $\mathcal{B}_{2}$, respectively, $\varepsilon>0$; there exists an operator $Q_{M, N, \varepsilon}: \mathcal{B}_{1}^{\prime} \rightarrow \mathcal{B}_{2}^{\prime}$ such that $\left\|Q_{M, N, \varepsilon}\right\| \leqslant C$ and the following two conditions are satisfied:

$$
\begin{aligned}
Q_{M, N, \varepsilon} T_{11} x & =0 \text { for each } x \in M, \\
\left\|Q_{M, N, \varepsilon} T_{12} y-T_{22} y\right\| & \leqslant \varepsilon \text { for each } y \in B_{N}
\end{aligned}
$$

(by $B_{\mathcal{B}}$ we denote the unit ball of a Banach space $\mathcal{B}$ ).

Let $X \in \mathcal{L}\left(\mathcal{B}_{1}, \mathcal{B}_{2}\right)$ be an operator of finite rank, such that

$$
\left.X\right|_{M}=0
$$

and

$$
X\left(B_{B_{1}}\right) \supset\left(\frac{C\left\|T_{11}\right\|}{\varepsilon}\right) B_{N} .
$$

(Here we use the assumption that the space $\mathcal{B}_{1}$ is infinite dimensional.)

Since $X$ is in the domain of $G_{T}$, there exists an operator $Q_{M, N, \varepsilon}$ such that $\left\|Q_{M, N, \varepsilon}\right\|$ $\leqslant C$ and

$$
Q_{M, N, \varepsilon}\left(T_{11}+T_{12} X\right)=T_{22} X
$$

or

$$
Q_{M, N, \varepsilon} T_{12} X-T_{22} X=-Q_{M, N, \varepsilon} T_{11}
$$


By (14) the condition (16) immediately implies (13).

Also (16) implies

$$
\left\|Q_{M, N, \varepsilon} T_{12} X-T_{22} X\right\| \leqslant C\left\|T_{11}\right\| .
$$

By condition (15) it implies

$$
\left(\frac{C\left\|T_{11}\right\|}{\varepsilon}\right)\left\|Q_{M, N, \varepsilon} T_{12} y-T_{22} y\right\| \leqslant C\left\|T_{11}\right\|
$$

for each $y \in B_{N}$. The condition (13) follows.

We endow the set of all triples $(M, N, \varepsilon)$ with the following ordering:

$$
\left(M_{1}, N_{1}, \varepsilon_{1}\right) \succ\left(M_{2}, N_{2}, \varepsilon_{2}\right) \text { if and only if } M_{1} \supset M_{2}, N_{1} \supset N_{2} \text {, and } \varepsilon_{1} \leqslant \varepsilon_{2} \text {. }
$$

Let $\mathcal{U}$ be an ultrafilter majorising this ordering. The set of all linear operators from $\mathcal{B}_{1}^{\prime}$ into $\left(\mathcal{B}_{2}^{\prime}\right)^{* *}$ with norm $\leqslant C$ is compact in the pointwise weak* topology. Hence the image of the ultrafilter $\mathcal{U}$ under the map $(M, N, \varepsilon) \mapsto Q_{M, N, \varepsilon}$ is convergent in this set. Let $\widetilde{Q}=w^{*}-\lim _{\mathcal{U}} Q_{M, N, \varepsilon}$ be the corresponding limit. Let $P:\left(\mathcal{B}_{2}^{\prime}\right)^{* *} \rightarrow \mathcal{B}_{2}^{\prime}$ be a bounded linear projection (whose existence is one of the conditions of Theorem 1). We let $Q=P \tilde{Q}$. It is easy to check that $Q T_{12}=T_{22}$ and $Q T_{11}=0$. By Proposition 1, the linear fractional relation $G_{T}$ is constant.

CASE 2. $T_{11}=0$. In this case the equation has the form: $Y T_{12} X=T_{22} X$. Take $X=u \otimes f$, then it is easy to see that $X \in \operatorname{dom} G_{T}$, if $T_{12} u \neq 0$. Hence for $T_{12} \neq 0, W(x)$ is dense in $\mathcal{B}_{2}$ and $\left\|T_{22} y\right\| \leqslant C\left\|T_{12} y\right\|$ for all $y \in \mathcal{B}_{2}$. This means that $T_{22}=Q T_{12}$, for some $Q \in \mathcal{L}\left(\mathcal{B}_{1}^{\prime}, \mathcal{B}_{2}^{\prime}\right)$, and $G_{T}$ is constant.

It remains to consider the case when both $T_{11}=0$ and $T_{12}=0$. In this case $T$ is of the form given in the statement of Theorem 1. It is easy to see that all relevant statements of Theorem 1 are valid. We have proved Theorem 1 in the case when $T_{21}=0$.

Now we consider the general case. Let $X_{0} \in \operatorname{dom} G_{T}$ and $Y_{0} \in G_{T}\left(X_{0}\right)$. Then

$$
T_{21}+T_{22} X_{0}=Y_{0}\left(T_{11}+T_{12} X_{0}\right) \text {. }
$$

Subtracting this equation from (3) and using simple algebraic transformations, we get

$$
\left(T_{22}-Y_{0} T_{12}\right)\left(X-X_{0}\right)=\left(Y-Y_{0}\right)\left(\left(T_{11}+T_{12} X_{0}\right)+T_{12}\left(X-X_{0}\right)\right) .
$$

Hence the equation (3) is equivalent to

$$
\widetilde{T}_{22} \tilde{X}=\tilde{Y}\left(\widetilde{T}_{11}+\tilde{T}_{12} \tilde{X}\right),
$$

where $\tilde{X}=X-X_{0}, \widetilde{Y}=Y-Y_{0}, \widetilde{T}_{12}=T_{12}, \widetilde{T}_{11}=T_{11}+T_{12} X_{0}$, and $\widetilde{T}_{22}=T_{22}-T_{12} Y_{0}$. The equation (17) describes $G_{\tilde{T}}$ for

$$
\tilde{T}=\left(\begin{array}{cc}
\tilde{T}_{11} & \widetilde{T}_{12} \\
0 & \tilde{T}_{22}
\end{array}\right) .
$$


It is clear that $0 \in \operatorname{dom} G_{\tilde{T}}$ and that $G_{\tilde{T}}$ is bounded. Therefore the argument above implies that either $G_{\tilde{T}}$ is constant, or $\widetilde{T}$ satisfies

$$
\tilde{T}_{11}=\tilde{T}_{12}=\tilde{T}_{21}=0
$$

It is easy to see that " $G_{\tilde{T}}$ is constant" implies " $G_{T}$ is constant", and that (18) implies that $T$ is of the form (7).

In particular, the result holds when $\mathcal{B}_{2}^{\prime}$ is a reflexive space.

The following lemma shows that in the reflexive case it is enough to require boundedness of $G_{T}$ on a weakly dense subspace in $\operatorname{dom} G_{T}$ only. (The basic facts about the weak operator topology and the strong operator topology which we use below can be found in [5, Chapter V].)

LEMma 1. Suppose that the space $\mathcal{B}_{2}^{\prime}$ is reflexive. A bounded linear fractional relation $G_{T}$ on a subspace $\mathcal{R}$ of $\mathcal{L}\left(\mathcal{B}_{1}, \mathcal{B}_{2}\right)$ can be extended to a bounded linear fractional relation on the closure $\mathcal{W}$ of $\mathcal{R}$ in the weak operator topology.

PROOF: Since the closures of a linear subspace in the weak operator topology and the strong operator topology coincide, for each $X \in \mathcal{W}$ there is a net $X_{\alpha} \in \mathcal{R}$ which converges to $X$ in the strong operator topology. Let $Y_{\alpha} \in G_{T}\left(X_{\alpha}\right)$; by the boundedness and reflexivity conditions we may assume that the net $Y_{\alpha}$ converges to some $Y \in \mathcal{W}$ in the weak operator topology. Then

$Y\left(T_{11}+T_{12} X\right)-\left(T_{21}+T_{22} X\right)=\left(Y-Y_{\alpha}\right)\left(T_{11}+T_{12} X\right)+Y_{\alpha} T_{12}\left(X-X_{\alpha}\right)+T_{22}\left(X_{\alpha}-X\right) \rightarrow 0$

in the weak operator topology.

At this moment it is not clear to what extent the restriction on $\mathcal{B}_{2}^{\prime}$ in Theorem 1 can be relaxed. Our next result shows that some restrictions on $\mathcal{B}_{2}^{\prime}$ are necessary for an analogue of the Liouville theorem for linear fractional relations to be valid. Below we assume that all direct sums are in $\ell_{\infty}$ sense. It means that $\|(x, y)\|=\max \{\|x\|,\|y\|\}$ for $(x, y) \in X \oplus Y$. Necessary background in Banach space theory can be found in [21].

THEOREM 2. If $\mathcal{B}_{1}=\mathcal{B}_{1}^{\prime}=\ell_{\infty} \oplus c_{0}$ and $\mathcal{B}_{2}=B_{2}^{\prime}=\ell_{\infty} \oplus c_{0} \oplus \ell_{1}(\Gamma)$, where $\Gamma$ has the cardinality of continuum, then there exists $T$ of the form (1) such that the linear fractional relation $G_{T}$ is bounded, $\operatorname{dom} G_{T}=\mathcal{L}\left(\mathcal{B}_{1}, \mathcal{B}_{2}\right)$, but $G_{T}$ is not constant.

PROOF: We need the following properties of the introduced objects

(1) The space $\mathcal{B}_{1}$ is isometric to $\mathcal{B}_{1} \oplus \mathcal{B}_{1}$ (because the direct sums are in $\ell_{\infty}$ sense).

(2) $\mathcal{B}_{1}$ is isometric to an uncomplemented subspace of $\mathcal{B}_{1}$. This fact immediately follows from the well-known fact: the canonical image of $c_{0}$ in $\ell_{\infty}$ is uncomplemented (see [21, Section II.4.n]). Let $A: \mathcal{B}_{1} \rightarrow \mathcal{B}_{1}$ be such an isometry. 
(3) Each operator from $\mathcal{B}_{1}$ into $\ell_{1}(\Gamma)$ is compact (see [21, Section I.1.b]).

(4) For each finite set $Z$ in $\mathcal{B}_{1}$ and each $\varepsilon>0$ there exists a finite-dimensional subspace $M$ in $\mathcal{B}_{1}$, which is isometric to $\ell_{\infty}^{n}$ for some $n \in \mathrm{N}$ and satisfies $\operatorname{dist}(z, M)<\varepsilon$ for each $z \in Z$ (see [21, Section II.5.b]).

(5) There exists a quotient map $\varphi: \ell_{1}(\Gamma) \rightarrow \mathcal{B}_{1}$ (see [21, p. 37]).

Now we define operators $T_{i j}$. In all these definitions we use representations:

$$
\begin{aligned}
& \mathcal{B}_{1}=\mathcal{B}_{1} \oplus \mathcal{B}_{1}, \\
& \mathcal{B}_{2}=\mathcal{B}_{1} \oplus \mathcal{B}_{1} \oplus \ell_{1}(\Gamma),
\end{aligned}
$$

where in (19) we use the existence of isometry from (1). When we write vectors of $\mathcal{B}_{1}$ as pairs, and vectors of $\mathcal{B}_{2}$ as triples, we mean the decompositions (19) and (19).

Let $T_{11}: \mathcal{B}_{1} \rightarrow \mathcal{B}_{1} \oplus \mathcal{B}_{1}$ be defined by $T_{11}(x)=(x, 0)$ (that is, $T_{11}$ is isometry of $\mathcal{B}_{1}$ onto its 'half').

$$
\begin{aligned}
& T_{12}\left(z_{1}, z_{2}, z_{3}\right)=\left(\varphi\left(z_{3}\right), A \varphi\left(z_{3}\right)\right), \\
& T_{22}\left(z_{1}, z_{2}, z_{3}\right)=\left(\varphi\left(z_{3}\right), \varphi\left(z_{3}\right), 0\right),
\end{aligned}
$$

and

$$
T_{21}(x)=(x, 0,0) .
$$

LEMma 2. The linear fractional relation $G_{T}$ is not constant.

Proof: Assume the contrary. By Proposition 1, there exists $Q \in \mathcal{L}\left(\mathcal{B}_{1}, \mathcal{B}_{2}\right)$ such that $Q T_{12}=T_{22}$ and $Q T_{11}=T_{21}$. By the definitions of $T_{11}$ and $T_{21}$, we get

$$
Q(x, 0)=(x, 0,0) \forall x \in \mathcal{B}_{1} .
$$

Using the definitions of $T_{12}$ and $T_{22}$, and the fact that $\varphi$ is a quotient map, we get

$$
Q(x, A x)=(x, x, 0)
$$

Therefore

$$
Q(0, A x)=(0, x, 0) .
$$

Consider $R: \mathcal{B}_{2} \rightarrow \mathcal{B}_{1}$ defined by

$$
R(u, x, z)=(0, A x) .
$$

Then $R Q$ is a projection of $\mathcal{B}_{1} \oplus \mathcal{B}_{1}$ onto $0 \oplus A \mathcal{B}_{1}$, the existence of such projection contradicts the fact that $A \mathcal{B}_{1}$ is uncomplemented in $\mathcal{B}_{1}$.

It remains to show that $\operatorname{dom} G_{T}=\mathcal{L}\left(\mathcal{B}_{1}, \mathcal{B}_{2}\right)$ and that $G_{T}$ is bounded. That is, we need to find for each $K: \mathcal{B}_{1} \rightarrow \mathcal{B}_{2}$ an operator $\alpha(K): \mathcal{B}_{1} \rightarrow \mathcal{B}_{2}$, such that

$$
\left(T_{21}+T_{22} K\right)=\alpha(K)\left(T_{11}+T_{12} K\right)
$$


and

$$
\sup _{K}\|\alpha(K)\|<\infty \text {. }
$$

Let $\dot{K} b=\left(K_{1} b, K_{2} b, K_{3} b\right)$ according to the three components of $\mathcal{B}_{2}$. The condition (19) can be written as

$$
\alpha(K)\left(b+\varphi\left(K_{3} b\right), A \varphi\left(K_{3} b\right)\right)=\left(b+\varphi\left(K_{3} b\right), \varphi\left(K_{3} b\right), 0\right)
$$

We need to establish the existence of such "moderate-norm" operator $\alpha(K)$ (no matter how large the norm of $K$ is).

The operator $\alpha(K)$ should map pairs (according to the decomposition (19)) onto triples (according to the decomposition (19)). It is easy to determine the first component of $\alpha(K)$, and to suggest the most natural third component, namely

$$
\alpha(K)(x, y)=(x, ?, 0)
$$

It remains to determine the operator which should replace the question mark.

The operator $K_{3}$ is compact by the condition (3) above. Hence, $A \varphi K_{3}\left(B_{\mathcal{B}_{1}}\right)$ is a compact set. Let $\left\{x_{i}\right\}_{i=1}^{n}$ be an $\varepsilon$-net in it. By the property (4) we can find a finitedimensional subspace $M \in \mathcal{B}_{1}$ isometric to $\ell_{\infty}^{m}$ for some $m$ such that $\operatorname{dist}\left(x_{i}, M\right)<\varepsilon \forall i$. Let $P$ be a projection of norm 1 onto $M$ and let $x \in A \varphi K_{3}\left(B_{B_{1}}\right)$. There exists $y \in M$ such that $\|x-y\| \leqslant 2 \varepsilon$. Hence $\|P x-P y\| \leqslant 2 \varepsilon$. Since $y=P y$, we get $\|x-P x\| \leqslant 4 \varepsilon$. In. other words

$$
\left\|(I-P) A \varphi K_{3}\right\| \leqslant 4 \varepsilon .
$$

On the other hand, it is clear that $P$ can be considered as an operator defined on the whole space $\mathcal{B}_{1} \oplus \mathcal{B}_{1}$, we let $P(x, 0)=0$. We consider an auxiliary operator $U: \mathcal{B}_{1} \oplus \mathcal{B}_{1} \rightarrow \mathcal{B}_{1} \oplus \mathcal{B}_{1}$ given by $U(x, y)=\left(x-A^{-1} P y, P y\right)$. The norm of this operator is $\leqslant 2$. Another useful property of this operator is that

$$
U\left(T_{11}+T_{12} K\right) b=\left(b+(I-\widetilde{P}) \varphi K_{3} b, P A \varphi K_{3} b\right),
$$

where $\widetilde{P}=A^{-1} P A$. The first operator in the right-hand side of $(20)$ is a small perturbation of the identity, namely

$$
(1-4 \varepsilon)\|b\| \leqslant\left\|b+(I-\widetilde{P}) \varphi K_{3} b\right\| \leqslant(1+4 \varepsilon)\|b\| .
$$

We let

$$
\alpha(K)(x, y)=\left(x, A^{-1} P y+D\left(I-A^{-1} P\right) x, 0\right)
$$

where $D$ is an operator satisfying

$$
D\left(b+(I-\widetilde{P}) \varphi K_{3} b\right)=\varphi K_{3} b-\tilde{P} \varphi K_{3} b
$$


Such a "moderate-norm" operator $D$ exists because of $(21)$ and because $(I-\widetilde{P}) \varphi K_{3}$ is a "moderate-norm" operator.

Our next purpose is to prove an analogue of Theorem 1 for linear fractional transformations $H_{T}: \mathcal{L}\left(\mathcal{B}_{1}, \mathcal{B}_{2}\right) \rightarrow \mathcal{L}\left(\mathcal{B}_{1}^{\prime}, \mathcal{B}_{2}^{\prime}\right)$ of the form

$$
H_{T}(X)=\left(T_{21}+T_{22} X\right)\left(T_{11}+T_{12} X\right)^{-1} .
$$

The domain of $H_{T}$ is defined by dom $H_{T}=\left\{X \in \mathcal{L}\left(\mathcal{B}_{1}, \mathcal{B}_{2}\right): T_{11}+T_{12} X\right.$ is invertible $\}$. It is clear that $\operatorname{dom} H_{T} \neq \mathcal{L}\left(\mathcal{B}_{1}, \mathcal{B}_{2}\right)$ for each $T$ with $T_{12} \neq 0$. For linear fractional transformations we prove the following analogue of Theorem 1 (without any restrictions on the geometry of Banach spaces involved).

THEOREM 3. If a linear fractional transformation $H_{T}$ is such that $\operatorname{dom} H_{T} \neq \emptyset$ and $H_{T}$ is bounded on dom $H_{T}$, then $H_{T}$ is constant.

Proof: It is convenient to start with a change of the variable. Suppose $X_{0} \in$ $\operatorname{dom} H_{T}$. Let

$$
Y=\left(X-X_{0}\right)\left(T_{11}+T_{12} X_{0}\right)^{-1}
$$

Then

$$
\begin{aligned}
H_{T}(X) & =\left(T_{21}+T_{22} X_{0}+T_{22}\left(X-X_{0}\right)\right)\left(T_{11}+T_{12} X_{0}+T_{12}\left(X-X_{0}\right)\right)^{-1} \\
& =\left(\left(T_{21}+T_{22} X_{0}\right)\left(T_{11}+T_{12} X_{0}\right)^{-1}+T_{22} Y\right)\left(I+T_{12} Y\right)^{-1}
\end{aligned}
$$

Hence $H_{T}(X)=H_{S}(Y)$, where $S$ is a matrix given by

$$
\left(\begin{array}{ll}
S_{11} & S_{12} \\
S_{21} & S_{22}
\end{array}\right)=\left(\begin{array}{cc}
I & T_{12} \\
H_{T}\left(X_{0}\right) & T_{22}
\end{array}\right) .
$$

Therefore

$$
H_{S}(Y)=\left(S_{21}+S_{22} Y\right)\left(I+S_{12} Y\right)^{-1} .
$$

We shall prove that $S_{22}=S_{21} S_{12}$. This equality immediately implies that $H_{S}$ and $H_{T}$ are constant.

If $S_{12}=0$, then $S_{22}$ should also be equal to 0 (otherwise $H_{S}(Y)$ is unbounded). So we suppose that $S_{12} \neq 0$.

Let $y \in \mathcal{B}_{2} \backslash \operatorname{ker} S_{12}$ and let $x=S_{12} y$. Let $e \in \mathcal{B}_{1}^{*}$ be such that $e(x)=\|x\|\|e\|=1$. For $Y=e \otimes y$ we have $Y x=y$ and $S_{12} Y=e \otimes x$, so $\left\|S_{12} Y\right\|=1, S_{12} Y x=x$.

Therefore the form of the denominator in (23) implies that $\lambda Y \in \operatorname{dom} H_{S}$ for $\lambda \in(-1,1)$. Hence

$$
\left\|S_{21} S_{12} y+\lambda S_{22} y\right\|=\left\|\left(S_{21}+\lambda S_{22} Y\right) x\right\| \leqslant k\left\|\left(I+\lambda S_{12} Y\right) x\right\|=k\|(1+\lambda) x\|,
$$


where

$$
k=\sup _{Y \in \operatorname{dom} H_{S}}\left\|H_{S}(Y)\right\| .
$$

Letting $\lambda \searrow-1$ we get $S_{21} S_{12} y=S_{22} y$.

Since $\mathcal{B}_{2} \backslash$ ker $S_{12}$ is dense in $\mathcal{B}_{2}$ (we have assumed that $S_{12} \neq 0$ ), the same is true for all $y \in \mathcal{B}_{2}$. Hence $S_{21} S_{12}=S_{22}$.

\section{LinEAR FRACTIONAL RELATIONS IN $C^{*}$-ALgEBRAS}

Our next purpose is to find analogues of the Liouville Theorem for linear fractional relations on Banach algebras. (Theorem 1 in the case $\mathcal{B}_{1}=\mathcal{B}_{2}=\mathcal{B}_{1}^{\prime}=\mathcal{B}_{2}^{\prime}$ may be considered as a result of this type.)

A $C^{*}$-algebra is called primitive if it has a faithful (= injective) irreducible representation. The class of primitive algebras is quite wide: it includes all simple algebras and many others.

Recall that the multiplier algebra $M(\mathcal{A})$ of a $C^{*}$-algebra $\mathcal{A}$ may be realised as a subalgebra of the universal enveloping von Neumann algebra $W(\mathcal{A})$ consisting of all $T \in W(\mathcal{A})$ such that $T \mathcal{A} \subset \mathcal{A}$ and $\mathcal{A} T \subset \mathcal{A}$. So any representation of $\mathcal{A}$ extends to $M(\mathcal{A})$.

We say that a $C^{*}$-algebra $\mathcal{A}$ has property (D) if any element of $\mathcal{A}$ is a product $A V$ where $A \in \mathcal{A}$ is non-negative and $V \in M(\mathcal{A})$ is invertible. The property (D) holds for the algebra $\mathcal{K}(\mathcal{H})$ of all compact operators and for its unital extension. Also it holds for all finite and all purely infinite von Neumann algebras.

THEOREM 4. Suppose that a primitive $C^{*}$-algebra $\mathcal{A}$ has the property (D). Then a bounded linear fractional relation defined at each point of $\mathcal{A}$ is constant.

Proof: Since $\mathcal{A}$ is primitive, we may realise it as an irreducible (hence dense in the weak operator topology) subalgebra of $\mathcal{L}(\mathcal{H})$. By Lemma $1, G_{T}$ extends to a bounded linear fractional relation on $\mathcal{L}(\mathcal{H})$. By Theorem 1 , there is $Q \in \mathcal{L}(\mathcal{H})$ such that $T_{21}=$ $Q T_{11}, T_{22}=Q T_{12}$. It remains to prove that such an operator $Q$ can be found in $\mathcal{A}$.

By our assumption, $T_{11}=N V$, where $N \geqslant 0$ and $V$ is an invertible element of $M(\mathcal{A})$. By the remark above, $V$ is an invertible element of $\mathcal{L}(\mathcal{H})$. We claim that for $X_{0}=T_{12}^{*} V$, the subspace $\mathrm{cl}\left(\left(T_{11}+T_{12} X_{0}\right) \mathcal{H}\right)$ contains both $T_{11} \mathcal{H}$ and $T_{12} \mathcal{H}$. Since $V$ is an invertible element of $\mathcal{L}(\mathcal{H})$, we have $\left(T_{11}+T_{12} X_{0}\right) \mathcal{H}=\left(N+T_{12} T_{12}^{*}\right) \mathcal{H}$. Hence the orthogonal complement to $\left(T_{11}+T_{12} X_{0}\right) \mathcal{H}$ is the kernel of $N+T_{12} T_{12}^{*}$, which coincides with the intersection of the kernels of $N$ and $T_{12} T_{12}^{*}$; the latter coincides with the kernel of $T_{12}^{*}$.

By definition, there is $Y_{0} \in G_{T}\left(X_{0}\right)$ such that $Y_{0}\left(T_{11}+T_{12} X_{0}\right)=T_{21}+T_{22} X_{0}$. Since the same is true for $Q$, we get that $Y_{0}$ coincides with $Q$ on the range of $T_{11}+T_{12} X_{0}$. Hence they coincide on the closure of this subspace. By the above, it follows that they coincide 
on $T_{11} \mathcal{H}$ and $T_{12} \mathcal{H}$. Hence $Y_{0} T_{11}=T_{21}$ and $Y_{0} T_{12}=T_{22}$. This means that $Y_{0} \in G_{T}(X)$ for each $X \in \mathcal{L}(\mathcal{H})$.

Now we consider the opposite extreme and prove an analogue of the Liouville theorem for linear fractional relations on commutative $C^{*}$-algebras, that is, on algebras of continuous functions on compacta. Let $\Omega$ be a compactum, by $C(\Omega)$ we denote the space of all continuous functions on $\Omega$ with the supremum norm.

THEOREM 5. Let $\mathcal{A}=C(\Omega)$, and let $T_{i j} \in \mathcal{A}(i, j=1,2)$. Suppose that for each $X \in \mathcal{A}$ there is $Y=Y_{X} \in \mathcal{A}$ such that

$$
Y_{X}\left(T_{11}+T_{12} X\right)=T_{21}+T_{22} X
$$

and

$$
\left\|Y_{X}\right\| \leqslant f(\|X\|) \text {, where } f(a)=o(a) \text { as } a \rightarrow \infty .
$$

Then there exists $Q \in \mathcal{A}$ such that $Q T_{12}=T_{22}, Q T_{11}=T_{21}$.

Proof: Consider functions $X_{n}(\omega) \equiv n, n=0,1,2, \ldots$ Let $Y_{n}=Y_{X_{n}}$. Then

$$
Y_{0} T_{11}=T_{21}
$$

For $n \geqslant 1$ we can rewrite the defining identity for $Y_{n}$ in the form

$$
Y_{n} T_{12}-T_{22}=-\frac{Y_{n}}{n} T_{11}+\frac{T_{21}}{n} .
$$

By the condition (24) we get

$$
\lim _{n \rightarrow \infty}\left(Y_{n}(\omega) T_{12}(\omega)-T_{22}(\omega)\right)=0 \forall \dot{\forall} \in \Omega .
$$

It follows that there exists a function $Y_{\infty}$ on $\Omega$ such that

$$
Y_{\infty}(\omega) T_{12}(\omega)=T_{22}(\omega)
$$

Observe that the argument above does not imply that $Y_{\infty}$ is continuous.

Let $Z_{i}=\left\{\dot{\omega}: T_{1 i}(\omega)=0\right\}, i=1,2$, and let $Z=Z_{1} \cap Z_{2}$.

We shall need the following observations.

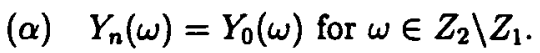

( $\beta) \quad Y_{n}(\omega)=Y_{\infty}(\omega)$ for $\omega \in Z_{1} \backslash Z_{2}, n \geqslant 1$.

Statements $(\alpha)$ and $(\beta)$ follow immediately from the definitions.

$(\gamma) \quad Y_{0}(\omega)=Y_{\infty}(\omega)$ for $\omega \in \Omega \backslash\left(Z_{1} \cup Z_{2}\right)$. 
Proof of $(\gamma)$ : Consider $\omega_{0} \in \Omega \backslash\left(Z_{1} \cup Z_{2}\right)$ and let $X$ be the constant function $-\left(T_{11}\left(\omega_{0}\right)\right) /\left(T_{12}\left(\omega_{0}\right)\right)$. Then

$$
Y_{X}(\omega)\left(T_{11}(\omega)-T_{12}(\omega) \cdot \frac{T_{11}\left(\omega_{0}\right)}{T_{12}\left(\omega_{0}\right)}\right)=T_{21}(\omega)-T_{22}(\omega) \cdot \frac{T_{11}\left(\omega_{0}\right)}{T_{12}\left(\omega_{0}\right)} .
$$

Evaluating both sides at $\omega_{0}$ we get

$$
0=T_{21}\left(\omega_{0}\right)-T_{22}\left(\omega_{0}\right) \cdot \frac{T_{11}\left(\omega_{0}\right)}{T_{12}\left(\omega_{0}\right)}
$$

Hence $Y_{\infty}\left(\omega_{0}\right)=Y_{0}\left(\omega_{0}\right)$.

Now we turn to definition of $Q$. Observe that the equations (25) and (26) imply that $T_{11}(\omega)=T_{12}(\omega)=T_{21}(\omega)=T_{22}(\omega)=0$ for $\omega \in Z$. Since $\Omega$ is compact (and hence each continuous function on its closed subset has a continuous extension to $\Omega$ ), it is enough to define $Q$ satisfying the conditions of Theorem 5 on $\operatorname{cl}(\Omega \backslash Z)$.

We let

$$
Q(\omega)=\left\{\begin{array}{l}
\frac{T_{21}(\omega)}{T_{11}(\omega)}=Y_{0}(\omega) \text { for } \omega \notin Z_{1} \\
\frac{T_{22}(\omega)}{T_{12}(\omega)}=Y_{\infty}(\omega) \text { for } \omega \in\left(Z_{1} \backslash Z_{2}\right)
\end{array}\right.
$$

and try to extend it to $\operatorname{cl}(\Omega \backslash Z)$.

This approach does not work if and only if there exists a point $\alpha \in \operatorname{cl}(\Omega \backslash Z)$ such that $\lim _{\mathcal{U}_{a}} Q$ does not exist, where $\mathcal{U}_{\alpha}$ is the filter on $\Omega \backslash Z$ given by

$$
\mathcal{U}_{a}=\{U \backslash Z: U \text { is a neighbourhood of } \alpha\}
$$

This, in turn, can happen if and only if one of the following is true

(1) There exists an ultrafilter $\mathcal{V}$ majorising $\mathcal{U}_{\alpha}$ such that $\lim _{\nu}|Q|=\infty$ or;

(2) There exist two ultrafilters $\mathcal{V}$ and $\mathcal{W}$ majorising $\mathcal{U}_{\alpha}$ such that

$$
\lim _{\mathcal{V}} Q \neq \lim _{\mathcal{W}} Q
$$

In the case (1) we may assume that $Z_{1} \backslash Z_{2} \in \mathcal{V}$. Hence we get for $Y_{1}$ (defined above)

$$
\lim _{\mathcal{V}} Y_{1}=\lim _{\mathcal{V}} \frac{T_{22}}{T_{12}}=\lim _{\mathcal{V}} Q
$$

A contradiction.

As for (2): without loss of generality we may assume that one of the following is true
(a) $Z_{1} \backslash Z_{2}$ belongs to both $\mathcal{V}$ and $\mathcal{W}$.
(b) $Z_{1} \backslash Z_{2} \in \mathcal{V}$ and $\Omega \backslash Z_{1} \in \mathcal{W}$. 
In the case (a) we get a contradiction in a straightforward way: it implies that $\lim _{\mathcal{V}} Y_{1} \neq \lim _{W} Y_{1}$, this contradicts the continuity of $Y_{1}$.

In the case (b), let

$$
K_{n}=\left\{\omega: T_{11}(\omega)+T_{12}(\omega) n \neq 0\right\} .
$$

The statement $(\gamma)$ implies that $Y_{n}(\omega)=Y_{0}(\omega)=Y_{\infty}(\omega)$ provided $\omega \in K_{n} \cap\left(\Omega \backslash\left(Z_{1} \cup Z_{2}\right)\right)$.

The case (b) contains the following subcases

$$
\text { (bo) } Z_{2} \backslash Z_{1} \in \mathcal{W} \text {. }
$$

In this case

$$
\lim _{\mathcal{W}} Y_{1}=\lim _{\mathcal{W}} \frac{T_{21}}{T_{11}}=\lim _{\mathcal{W}} Q \neq \lim _{\mathcal{V}} Q=\lim _{\mathcal{V}} \frac{T_{22}}{T_{12}}=\lim _{\mathcal{V}} Y_{1},
$$

a contradiction.

$$
\left(\mathrm{b}_{n}\right) \quad n \geqslant 1 . K_{n} \cap\left(\Omega \backslash\left(Z_{1} \cup Z_{2}\right)\right) \in \mathcal{W} .
$$

In such a case, by the observation above

$$
\lim _{\mathcal{W}} Y_{n}=\lim _{\mathcal{W}} Q \neq \lim _{\mathcal{V}} Q=\lim _{\mathcal{V}} \frac{T_{22}}{T_{12}}=\lim _{\mathcal{V}} Y_{n}
$$

a contradiction.

It remains to show that at least one of the cases $\left(b_{i}\right)(i=0,1,2)$ occurs. Assume the contrary. Using the basic properties of ultrafilters we get that $\mathcal{W}$ contains

$$
\left(\Omega \backslash\left(Z_{1} \cup Z_{2}\right)\right) \cap\left\{\omega: T_{11}(\omega)+T_{12}(\omega)=0\right\}
$$

and

$$
\left(\Omega \backslash\left(Z_{1} \cup Z_{2}\right)\right) \cap\left\{\omega: T_{11}(\omega)+T_{12}(\omega) \cdot 2=0\right\} .
$$

Hence $\mathcal{W}$ contains

$$
\left(\Omega \backslash\left(Z_{1} \cup Z_{2}\right)\right) \cap\left\{\omega: T_{11}(\omega)+T_{12}(\omega)=0\right\} \cap\left\{\omega: T_{11}(\omega)+T_{12}(\omega) \cdot 2=0\right\} .
$$

Since this set is empty, we get a contradiction.

REMARK. The result extends to many other Banach algebras of functions, for example the algebra $A(D)$ of bounded continuous functions on a compact $D \subset \mathrm{C}^{n}$ analytic in all inner points of $D$. For the proof it suffices to apply Theorem 5 and to note that if a continuous function $f$ coincides with an analytic function $g$ outside the nullset of a non-zero analytic function, then $f=g$. 


\section{REFERENCES}

[1] D. Alpay and V. Khatskevich, 'Linear fractional transformations: basic properties, applications to spaces of analytic functions and Schroeder's equation', Int. J. Appl. Math. 2 (2000), 459-476.

[2] T.Ya. Azizov and I.S. Iokhvidov, Linear operators in spaces with an indefinite metric (John Wiley and Sons, Chichester, 1989).

[3] C.C. Cowen, 'Iteration and the solution of functional equations for functions analytic in the unit disk', Trans. Amer. Math. Soc. 265 (1981), 69-95.

[4] C.C. Cowen and B.D. MacCluer, 'Linear fractional maps of the ball and their composition operators', Acta Sci. Math. (Szeged) 66 (2000), 351-376.

[5] N. Dunford and J.T. Schwartz, Linear operators, Part I: General Theory (Interscience Publishers, New York, 1958).

[6] M. Elin and V. Khatskevich, 'Triangular plus-operators in Banach spaces: applications to the Koenigs embedding problem', J. Nonlinear Convex Anal. 6 (2005), 173-185.

[7] J.W. Helton, 'Operators unitary in an indefinite metric and linear fractional transformations', Acta Sci. Math. (Szeged) 32 (1971), 261-266.

[8] I.S. Iohvidov, M.G. KreIn and H. Langer, Introduction to the spectral theory of operators in spaces with an indefinite metric (Akademie-Verlag, Berlin, 1982).

[9] V.A. Khatskevich, 'Fixed points of generalized linear-fractional transformations', (in Russian), Izv. Akad. Nauk SSSR Ser. Mat. 39 (1975), 1130-1141.

[10] V. Khatskevich, 'Some global properties of fractional-linear transformations', in Nonselfadjoint operators and related topics (Beer Sheva, 1992), Oper. Theory Adv. Appl. 73 (Birkhäuser, Basel, 1994), pp. 355-361.

[11] V. Khatskevich, 'Generalized fractional linear transformations: convexity and compactness of the image and the pre-image; applications', Studia Math. 137 (1999), 169-175.

[12] V.A. Khatskevich, M.I. Ostrovskii and V.S. Shulman, 'Linear fractional relations for Hilbert space operators', Math. Nachrichten (to appear).

[13] V. Khatskevich, S. Reich and D. Shoikhet, 'One-parameter semigroups of fractional-linear transformations', in Operator theory, system theory and related topics (Beer-Sheva/Rehovot, 1997), Oper. Theory Adv. Appl. 123 (Birkhäuser, Basel, 2001), pp. 401-411.

[14] V. Khatskevich, S. Reich and D. Shoikhet, 'Abel-Schröder equations for linear fractional mappings and the Koenigs embedding problem', Acta Sci. Math. (Szeged) 69 (2003), 67-98.

[15] V. Khatskevich and V. Senderov, 'Basic properties of linear fractional mappings of operator balls: Schroeder's equation', in Operator theory and its applications (Winnipeg, $M B$, 1998), Fields Inst. Commun. 25 (Amer. Math. Soc., Providence, RI, 2000), pp. 331-344.

[16] M.G. Krein, 'On an application of the fixed point principle in the theory of linear transformations of spaces with an indefinite metric', (in Russian), Uspehi Matem. Nauk (N.S.) 36 (1950), 180-190.

[17] M.G. Krein, 'A new application of the fixed-point principle in the theory of operators in a space with indefinite metric', (in Russian), Dokl. Akad. Nauk SSSR 154 (1964), 1023-1026. 
[18] M.G. Krein and J.L. Smuljan, 'Plus-operators in a space with indefinite metric', (in Russian), Matem. Issled. 1 (1966), 131-161. English translation Amer. Math. Soc. Transl. 85 (1969), 93-113.

[19] M.G. Krein and J.L. Smuljan, ' $\mathcal{J}$-polar representation of plus-operators', (in Russian), Matem. Issled. 1 (1966), 172-210. English translation Amer. Math. Soc. Transl. 85 (1969), 115-143.

[20] M.G. Krein and J.L. Smuljan, 'On linear-fractional transformations with operator coefficients', (in Russian), Matem. Issled. 2 (1967), 64-96. English translation Amer. Math. Soc. Transl. 103 (1974), 125-152.

[21] J. Lindenstrauss and L. Tzafriri, Classical Banach spaces, Lecture Notes in Math. 338 (Springer-Verlag, Berlin, 1973).

[22] E. Vesentini, 'Semigroups of linear contractions for an indefinite metric', Atti. Acad. Naz. Lincei Cl. Sci. Fis. Mat. Natur. Mem. (9) Mat. Appl. 2 (1994), 53-83.

Department of Mathematics

ORT Braude College

College Campus, P.O. Box, 78

Karmiel 21982

ISRAEL

e-mail: victor_kh@hotmail.com
Department of Mathematics and Computer Science St.-John's University 8000 Utopia Parkway Queens, NY 11439 United States of America e-mail: ostrovsm@stjohns.edu

Department of Mathematics Vologda State Technical University 15 Lenina str.

Vologda 160000

Russia

e-mail: shulman_v@yahoo.com 